Structuration de l'espace énonciatif et appropriation du discours stéréotypé dans le conte populaire transcrit

\title{
Christine Copy
}

\section{(2) OpenEdition Journals \\ Édition électronique \\ URL : http://journals.openedition.org/esa/2299 \\ DOI : 10.4000/esa.2299 \\ ISSN : 2650-2623 \\ Éditeur \\ Société de stylistique anglaise}

Édition imprimée

Date de publication : 31 décembre 2010

Pagination : 73-90

ISSN : 2116-1747

\section{Référence électronique}

Christine Copy, «Structuration de l'espace énonciatif et appropriation du discours stéréotypé dans le conte populaire transcrit », Études de stylistique anglaise [En ligne], 1 | 2010, mis en ligne le 25 novembre 2018, consulté le 22 avril 2019. URL : http://journals.openedition.org/esa/2299 ; DOI : 10.4000/esa.2299 


\title{
STRUCTURATION DE L'ESPACE ÉNONCIATIF ET APPROPRIATION DU DISCOURS STÉRÉOTYPÉ DANS LE CONTE POPULAIRE TRANSCRIT
}

\author{
Christine Copy \\ Université de Pau et des Pays de l'Adour, EA 3958, IMAGER (LIDIL)
}

\begin{abstract}
:
In this article, the text-type markers of transcribed folktales are analysed as fixed expressions. Considering the way those expressions function in context, it is argued that i) fixedness is the sign of the commitment of a generic enunciative source called proto-enunciator and related to what Maingueneau (2004) calls hyperenunciation (hyperénonciation) ; ii) variations on text-type markers function as a way to posit a specific subjective source as well as they highlight the analytical reading necessary to interpret these expressions.
\end{abstract}

Keywords: text-type marker, fixed expression, variation, proto-enunciator, hyperenunciation, commitment

Le conte populaire transcrit est immédiatement reconnaissable et situé dans l'espace textuel par un ensemble de constructions linguistiques typiques qui présentent un haut degré de figement. Cette caractéristique qui le constitue formellement en genre, fait du conte, au-delà même des spécificités diégétiques de ce type de récit, un stéréotype linguistique et un récit stéréotypé dont les marqueurs typologiques comme once upon a time sont la partie visible.

L'existence de contraintes linguistiques fortes dans le texte a des conséquences importantes à plusieurs niveaux de la composition discursive.

D'un point de vue narratif par exemple, la mise en place d'une voix narrative est soumise à la contrainte des phrases toutes faites et la voix du conteur se trouve filtrée par un texte façonné d'avance. L'actualisation du récit réside alors dans l'appropriation qu'il fera du texte. La tension qui s'organise 
entre ces deux pôles figure l'espace narratif du conte et se noue tout particulièrement dans la situation de contage telle qu'elle est représentée dans les phrases d'introduction du conte transcrit.

D'un point de vue cognitif ensuite, se pose la question du fonctionnement en langue de ces expressions qui ont des points communs avec les expressions figées, au niveau formel mais aussi interprétatif, notamment une certaine opacité qui suppose une lecture dite non-compositionnelle.

D'un point de vue énonciatif également, dans la mesure où dans les expressions figées, les sources subjectives ne sont pas clairement définies. Lorsque ces expressions sont incluses dans un récit, l'autorité du narrateur est alors à démontrer dans un contexte de prise en charge partagée et d'énonciation complexe.

Et enfin d'un point de vue énonciatif, on peut se demander comment cette source subjective s'inscrit au niveau des marqueurs, mais également, quelles sont les stratégies mises en place pour inscrire dans l'énoncé un sujeténonciateur, dans le sens qu'on lui donne dans la Théorie des Opérations Énonciatives (dorénavant TOE), c'est-à-dire un support de point de vue repérable à partir de traces qu'il laisse dans l'énoncé.

C'est de l'ensemble de ces points que je vais traiter dans cet article.

\section{Expressions de début de contes et construction de l'espace narratif du récit}

Dans le contage oral, la configuration énonciative a trois composantes : d'une part, le conteur-narrateur et son auditoire qui se font face et interagissent au moment de la performance 1 en cours et d'autre part, l'autorité ancestrale garante des valeurs de validité du récit et de sa persistance à travers le temps. Dans le conte transcrit, la configuration intersubjective entre conteur et auditoire n'est plus immédiate mais représentée par l'intrication d'énoncés qui ont des coordonnées énonciatives sensiblement différentes.

L'exemple (1) en est une illustration :

(1) A long, long time ago-if I were there then, I wouldn't be there now; if I were there now and at that time, I would have a new story or an old story, or I might have no story at allthere was a king and a queen in Ireland, and they were married. (Folktales of Ireland)

L'intrication entre énoncés figurant les interventions d'un conteurnarrateur à la première personne (la fiction secondaire) et énoncés de l'histoire

1 Les termes de performance, qui est un anglicisme, et de contage sont utilisés par les spécialistes du conte oral pour renvoyer au moment où se dit le conte en public (Bricout 1992, Belmont 1997). 
narrée (la fiction primaire) ${ }^{2}$ à la troisième personne permet d'installer la relation entre situation de contage et situation du conte ${ }^{3}$. Cette intrication fait également apparaître une dimension textuelle importante dans le fonctionnement fictionnel du conte qu'est l'alternance entre :

- des énoncés extrêmement codifiés, à disposition, qui signalent clairement le registre du conte et qui transcendent l'unicité du récit puisqu'elles valent pour tous les récits du genre (once upon a time);

- des prises de parole du conteur alors qu'il semble chercher à s'écarter des chemins parfaitement balisés d'une entrée en matière fortement attendue.

Dans le cas des énoncés de débuts de contes, ce jeu énonciatif instaure une tension entre d'une part, unicité du contage qui marque la prise en charge du récit par une instance spécifique, le conteur particulier, et d'autre part, généricité ${ }^{4}$ du conte, perceptible dans le figement caractéristique des formules de début de conte.

Les expressions typiques des débuts de contes du type once upon a time/ il était une fois ont un rôle majeur dans la grammaire du genre et il suffit de les prononcer pour que soit convoqué l'univers du récit.

Dans les études consacrées au sujet, ces formules de débuts de contes sont en général considérées dans leur rôle typologique.

Virtanen (1992), par exemple, parle, pour once upon a time, de marqueur typologique (text-type marker) qui renvoient à la stratégie textuelle temporelle (text strategy) dominante du texte 5 .

Dans son travail sur la typologie textuelle, Adam (2001) souligne la signification énonciative de l'expression. Il lui attribue notamment un rôle de « mise à distance » opérée dans la dimension sémantique globale du texte : en

2 Voir Vuillaume (1990) pour qui l'histoire narrée dans le récit constitue la fiction primaire et les interventions du narrateur relevant d'un ancrage situationnel différent, la fiction secondaire.

3 L'adéquation du modèle que représentent les contes transcrits avec la réalité du contage oral est remise en cause par certains spécialistes du conte oral (voir Bricout 1992, Belmont 1997, entre autres). Pour ces auteurs, les contes transcrits ne seraient qu'une des versions possibles du texte reflétant les choix et parfois les opinions du transcripteur. Par ailleurs, la représentation du contage dans ces textes relèverait aussi d'une vision stéréotypée de celui-ci. En tout état de cause, le présent travail portant uniquement sur un corpus de contes transcrits, je ne me prononce pas sur les caractéristiques de la situation d'énonciation dans le conte oral, mais sur sa représentation dans le conte transcrit.

4 Le terme est ici à comprendre dans le sens de genre et de générique.

5 Pour Virtanen (1992), la stratégie textuelle est dénotée par le type et la récurrence des circonstants en début d'unités phrastiques et d'unités textuelles (high-level section). Dans le cas du conte, la stratégie textuelle est donc, d'après elle, temporelle. Il n'y a pas ici de traitement de la dimension opaque de ces marqueurs typologiques. 
introduisant son récit par il était une fois, le narrateur le place résolument dans le monde de la fiction et dirige la lecture en ce sens 6 .

Ces deux propositions, celle d'Adam et celle de Virtanen, s'accordent sur le fait que les énoncés formulaïques des débuts de contes sont à considérer comme des marqueurs typologiques. Cependant, ces analyses ne s'appuient pas sur les mêmes critères.

Pour Virtanen, il s'agit de décrire la mise en place d'une stratégie textuelle temporelle et elle donne donc une interprétation littérale de l'expression once upon a time en tant qu'élément adverbial.

Pour Adam, en revanche, c'est la valeur illocutoire supposée de l'expression qui est mise en avant.

Dans les deux cas cependant l'expression formulaïque est traitée comme un bloc, sans qu'il y ait de proposition d'analyse des éléments qui la composent.

Cette façon de caractériser ces expressions revient en fait à les considérer comme des expressions figées.

\section{Les marqueurs typologiques sont-ils des expressions figées?}

On peut en effet considérer que les expressions de débuts de contes ont beaucoup en commun avec les expressions figées et les proverbes et notamment une forme de non-compositionnalité corollaire de l'opacité référentielle et sémantique de ces formes discursives.

\section{Caractéristiques des expressions figées}

Parmi les critères souvent avancés pour définir les locutions ou expressions figées mais aussi les proverbes, on trouve notamment celui de noncompositionnalité. C'est ainsi, par exemple, que les décrit Martin (1997) qui en fait un critère définitoire :

[...] une locution prototypique est caractérisée [...] par sa non-compositionnalité. On a beau comprendre tous les mots qui entrent dans tirer le diable par la queue, cela ne suffit pas pour comprendre ce que cette locution veut dire (Martin 293).

De cette non-compositionnalité découlent des caractéristiques qui distinguent les expressions figées des énoncés construits en langue et dont on peut citer les suivantes :

6 «En commençant une narration par 'Il était une fois...', le narrateur opère une mise à distance à la fois énonciative [...] et fictionnelle [...], il donne au lecteur / auditeur une instruction sur l'ancrage énonciatif non actuel de ce qui suit et sur le monde singulier, non conforme aux lois qui régissent notre univers de référence, dans lequel il conviendra d'évaluer les faits relatés ». (Adam 2001, 24). C'est moi qui souligne. 
- Il n'y a pas d'axe paradigmatique :

(2) \#Qui roupille dîne (exemple de Kleiber 2004)

Ainsi, pour reprendre l'exemple de Kleiber, on ne peut pas faire permuter un des lexèmes de la locution avec un lexème appartenant au même champ lexical, sauf à faire un trait d'humour ce qui fait évoluer le proverbe originel vers un autre type d'énoncé.

- Il suffit de prononcer une partie seulement d'un proverbe pour que l'ensemble de la phrase soit entendu :

(3) "China, by contrast, has the ability to make regulatory and bureaucratic changes. They can do that quickly when there's a will."7 (Financial Time, 16/06/10)

Le caractère prédictible des éléments des expressions collocationnelles les distinguent ainsi des énoncés libres.

- Les proverbes et les locutions ont un fonctionnement en langue proche des lexies.

Outre le fait qu'elles font l'objet de recueils et de dictionnaires au même titre que les lexies, les expressions figées posent des problèmes d'interprétation aux apprenants mais aussi aux enfants qui en proposent en général une lecture littérale. Ceci tend à montrer que les expressions figées s'apprennent comme le lexique et que seule une connaissance préalable permet de maintenir ces énoncés dans le giron proverbial. Cette dimension qui repose sur l'apprentissage et sur la mémorisation est fondamentale dans l'appropriation des locutions.

Pour Grunig (1997), par exemple, ce dernier critère est même le seul valable pour définir les locutions. Ainsi le fonctionnement de la locution relèverait uniquement du domaine de la psycholinguistique et n'importe quelle phrase « peut acquérir un statut de titre, ou de phrase historique, ou de rituel, ou - à peu de choses près - même de proverbe, à condition d'avoir un statut social solidaire d'une inscription mémorielle ou d'avoir un taux de répétition ou de notoriété dans une circulation langagière qui les ait transformés en inscriptions mémorielles. » (235). Le cas de la locution ne tirez pas sur l'ambulance en français appuie cette hypothèse ${ }^{8}$.

Cette dimension collective et mémorielle soulevée par Grunig met l'accent sur le rôle des locutions dans l'appropriation par un locuteur de sa langue dans sa dimension partagée. Cette dimension signe en effet la

\footnotetext{
7 When there's a will, there's a way.

8 En effet, l'expression est relativement récente en français et a été utilisée pour la première fois dans un article de presse par Françoise Giroud.
} 
particularité énonciative de ces phrases, que Maingueneau (2004) a théorisée, dans une perspective polyphonique, à l'aide du concept de «particitation ».

\section{Les expressions figées comme énoncés de particitation}

Maingueneau (2004, 112-113) définit les énoncés de particitation selon deux critères essentiels qui sont : l'autonomie de l'énoncé et l'appartenance à un Thésaurus.

On parle d'autonomie de l'énoncé de particitation dans la mesure où il est reconnu comme une citation sans que ce caractère soit signalé par un verbe introducteur ou par une incise. L'énoncé est alors seulement «marqué par un décalage interne à l'énonciation» $(2004,110)$ et restitué dans son signifiant, même si une part de variation est parfois possible.

Cette constance du signifiant donne notamment aux proverbes un statut particulier au sein des phrases qui les incluent et participe à la construction de leur autonomie syntaxique qui est une de leurs propriétés définitoires. En effet, dans les cas d'enchâssement, les proverbes ne sont pas dépendants des mêmes coordonnées énonciatives que la partie enchâssante de l'énoncé ce qui rend problématique leur insertion dans le discours indirect (DI) et on hésite par exemple entre (4) et (5) :

(4) ?He said that a friend in need is a friend indeed.

(5) \#He said that a friend in need was a friend indeed.

Ainsi, en (4), l'énoncé est considéré comme fautif selon la norme car le verbe de la complétive reste au présent. En revanche, en (5), le prétérit ancre les coordonnées de la situation de l'énoncé rapporté dans celle de l'énoncé rapportant, ce qui pose problème quant à la valeur générique du proverbe et qui relève déjà d'un processus de défigement.

Cette caractéristique montre que l'autonomie syntaxique de ces énoncés est le corollaire d'une autonomie énonciative : la relation prédicative n'est pas repérée par rapport à la Situation de l'événement construite par l'énoncé dans lequel le proverbe est inclus et, pour garder pleinement son statut discursif, il doit rester au présent dans les phrases au passé. À ce titre, les proverbes font partie des énoncés que l'on ne peut pas, en tant que tel, mettre au discours indirect et qui restent repérés uniquement par rapport à la situation originelle de profération 9 .

Cette autonomie énonciative est également marquée par les particularités prosodiques de ce type d'expression: dans le discours, les proverbes sont

9 Ce qui les fait rejoindre de façon assez paradoxale d'autres types d'énoncés tels que les vocatifs, les exclamatifs, les insultes, qui ne sont pas non plus traduisibles en DI pour reprendre le terme de De Mattia (2000) et qui restent toujours repérés par rapport à la situation d'énonciation origine de profération. 
marqués par des ruptures intonatives et des schémas prosodiques bien particuliers.

Par ailleurs, les énoncés de particitation appartiennent à un Thésaurus défini comme un ensemble «d'énoncés aux contours plus ou moins flous » qui circule dans une communauté dont il est indissociable. Les deux, Thésaurus et communauté correspondante sont "référés» à un hyperénonciateur dont l'autorité garantit la validité de l'énoncé, c'est-à-dire son "adéquation aux valeurs, aux fondements d'une collectivité » (Maingueneau 2004, 112-113).

Les proverbes, les expressions figées relèvent du régime de la particitation, et Maingueneau y inclut le conte merveilleux qui fait plus particulièrement partie de ce qu'il appelle "les particitations de communion » (id., 121). De la même façon, on peut y inclure les marqueurs typologiques de début et de fin de contes.

En effet, les expressions de début de conte partagent les caractéristiques des expressions figées évoquées ici :

- Toute modification implique une perte, ou en tout cas, un passage vers autre chose, comme dans ce titre de dessin animé Twice upon a time;

- Elles peuvent être laissées en suspens : il suffit de dire once upon... pour que l'ensemble l'expression soit récupérée ;

- Elles sont marquées prosodiquement : la première phrase des contes fait l'objet d'un schéma intonatif spécifique qui, à l'oral, participe à la mise en place de l'univers du discours spécifique au récit.

- Elles relèvent bien de la particitation qui fonde leur valeur énonciative : dire once upon a time, c'est se constituer partie d'un ensemble flou de locuteurs qui constitue une instance énonciative dont on reconnaît l'autorité ;

- Elles font l'objet d'une mémorisation qui commence dès l'enfance avec la lecture des contes.

Ce qui revient à dire que le sens de l'expression once upon a time n'est pas égal à la somme de once + upon $+a+$ time et que son interprétation échappe en partie à une analyse compositionnelle.

Cependant, l'analyse non-compositionnelle des expressions figées ne suffit pas à rendre compte du phénomène dans son ensemble. En effet, même si les critères de définition des expressions figées posent problème, il y a paradoxalement un certain consensus sur ce que sont les expressions figées et proverbiales: les locuteurs natifs et adultes sont en général d'accord pour attribuer l'étiquette de proverbe ou expression figée, même s'il n'est pas toujours évident d'en déterminer le sens et même s'ils ne l'ont jamais entendu. Ainsi, bien qu'ils ne suffisent pas à définir ce qu'est un proverbe ou une locution, certains schémas récurrents fondés sur des régularités linguistiques, 
syntaxiques, prosodiques ou encore sémantiques facilitent leur entrée dans le Thésaurus et montrent que tout syntagme n'est pas un candidat éligible. Cette particularité laisse supposer l'existence d'une compétence linguistique du proverbe et des expressions figées, compétence qui serait acquise pendant l'apprentissage du langage et qui aurait bien comme ressort les propriétés spécifiques à la langue.

Par ailleurs, le sens de ces expressions peut être déduit, au moins en partie, à partir du sens des marqueurs qu'on y trouve. Ainsi, on ne peut pas nier que dans once upon a time il y a aussi once+upon $+a+$ time et qu'on entend bien la détermination à la fois quantitative (once qui renvoie à l'unicité de l'occurrence) et qualitative ( $a$ qui renvoie à la typicalité de l'occurrence) qui sert de repère à l'espace temporel du récit. Ainsi, le sens de l'expression est bien déduit en partie au moins à partir des éléments qui la composent.

En effet, sans cette appartenance à la langue ordinaire, aucune modification des expressions figées ne serait envisageable, ce qui est contredit par l'usage comme le montre la citation suivante :

(6) Paul Curry was back, in jeans and a sweatshirt and with two large pizzas Mona had made and sent so boys could be boys for the night. (Grisham, J. Bleachers).

La possibilité de faire fonctionner les marqueurs des expressions figées dans un axe paradigmatique montre que ces expressions conservent une certaine malléabilité et qu'elles sont en partie au moins analysées de manière compositionnelle au même titre que les énoncés libres de la langue.

\section{Défigement et analyse compositionnelle dans les expressions figées}

L'appartenance à la langue ordinaire des expressions figées a les implications suivantes :

- Les expressions figées sont analysables à partir du sens des lexèmes et des marqueurs qui les composent ;

- Les expressions figées sont un contexte pertinent pour déterminer la forme schématique de ces lexèmes et marqueurs.

- Il est possible de modifier les coordonnées énonciatives de ces énoncés vers un repérage par rapport à la Situationrepère de l'énonciation en cours.

Ainsi, la possibilité même de modifications dans les expressions figées est analysable comme la trace d'un calcul compositionnel qui existe déjà dans la lecture figée de l'expression. 


\section{Expressions figées et calcul compositionnel}

Grunig (1997) insiste sur ce point lorsqu'elle met en avant le fait que le critère de non-compositionnalité ne saurait être une caractéristique absolue des expressions figées et que, par ailleurs, toutes les locutions ne sont pas égales, si on peut dire, face à ce principe. Pour nombre de locutions, l'interprétation résulte en partie d'un calcul effectué en fonction du sens des parties : dans jeter l'argent par la fenêtre, par exemple, une partie du sens attribué à l'expression résulte bien d'une analyse compositionnelle, même s'il ne résulte pas d'une interprétation littérale.

Cependant, dans le cas des locutions, le calcul compositionnel n'est pas tout à fait du même ordre que dans l'usage de la langue dans la mesure où il apparaît comme secondaire, c'est-à-dire qu'il résulte d'une opération qui s'effectuerait dans un deuxième temps, pour reprendre la définition de Numberg et al. (1994) :

[...] compositionality - that is, the degree to which the phrasal meaning, once known, can be analyzed in terms of the contribution of the idiom parts. (Nunberg et al. 1994, 498)

Ce caractère ressort tout particulièrement dans les défigements qui font intervenir les variations possibles dans les locutions. Ces détournements les ramènent de fait dans la langue articulée à deux titres : i) ils permettent l'inscription d'une subjectivité dans l'énoncé ; ii) ils montrent que la valeur centrale des marqueurs en jeu joue un rôle dans l'interprétation des expressions figées.

\section{Défigement et subjectivité}

C'est l'analyse que fait Rastier (1994) dans son travail sur l'aspect sémantique du défigement. Pour lui, dans la plupart des cas de défigements «les parcours interprétatifs sont redoublés par une duplicité ludique ou satirique » (312). Le défigement représente alors une forme d'émancipation du locuteur par rapport aux règles strictes de la locution. Ainsi, précise Rastier " c'est sans doute par le défigement qu'un auteur peut au mieux manifester sa liberté » (310). Cependant, souligne-t-il, « les jeux de mots qui président aux défigements mettent en évidence des phénomènes normaux et ordinaires pour la sémantique contextuelle. » (310).

Dans le cadre d'une analyse énonciative, les modifications opérées dans les expressions figées sont analysables comme les traces de l'inscription d'un sujet-énonciateur dans l'énoncé. Dans une description relevant du cadre de la $T O E$, l'inscription du sujet-énonciateur se fait à tous les niveaux de la constitution de l'énoncé. Au niveau prédicatif, le sujet-énonciateur est responsable des choix des notions instanciant la lexis, de l'orientation de la 
relation prédicative ainsi que du choix d'une polarité de l'énoncé (positive ou négative). Au niveau énonciatif, l'inscription d'un sujet-énonciateur est marquée par le choix des déterminations temporelles, aspectuelles et modales mais aussi des marqueurs de détermination nominale et des déictiques. Ces déterminations sont les traces d'un repérage par rapport à un système situationnel qui peut comprendre plusieurs rangs de repérage selon que l'on a affaire à une énonciation directe ou indirecte.

Dans les expressions figées, aucune modification permettant d'inscrire une subjectivité n'est a priori acceptable. Ainsi, l'ensemble des choix mentionnés plus haut est indépendant de l'énonciation en cours. De ce fait, la prise en charge modale et aspectuo-temporelle de l'énoncé s'effectue par rapport à une entité comprise comme une forme d'énonciateur rapporté. Ce point est illustré par l'exemple suivant où le proverbe a friend in need is a friend indeed se trouve inséré dans du DI :

(7) Whoever first said that "a friend in need is a friend indeed" certainly knew what he (or she) was talking about. http://www.womensweb.ca/violence/dv/articles.php

En (7), bien que THAT marque l'enchâssement de la complétive dans la principale, et donc le repérage de l'énoncé rapporté par rapport aux coordonnées de l'énoncé rapportant ${ }^{10}$, l'insertion du proverbe dans le discours rapportant reste partielle. L'autonomie que conserve l'énoncé est marquée d'une part par la présence de guillemets que signale un décalage entre la situation-repère origine de l'énoncé enchâssant et celle de l'énoncé enchâssé et d'autre part par le maintien du verbe au présent simple. Ce maintien de la détermination verbale du proverbe permet de le conserver dans le giron proverbial et marque le repérage par rapport à une entité décrochée de l'énonciation en cours.

D'un point de vue énonciative, où la source de prise en charge est décrite à partir de repérages dont les marqueurs sont la trace, cela revient à décrire le figement en tant que marqueur dans le sens culiolien du terme, c'est-à-dire trace dans l'énoncé d'une source énonciative correspondant à un point de vue subjectif. Dans la mesure où, dans les expressions figées, ce point de vue doit être celui de tout énonciateur potentiel, il est fortement stabilisé et renvoie à une entité de type proto-énonciateur et qui a la spécificité d'être transsubjectif ${ }^{11}$.

10 Pour une analyse énonciative de THAT en DI, voir De Mattia $(2000,109 s q$.) et Hanote \& Chuquet $(2004,59 s q)$

11 Cette source proto-énonciative assure notamment la prise en charge dans les inversions locative, dont Gournay (2005, 2006 entre autres) a montré que toute variation modale de la prédication était impossible, ce qu'elle a décrit en termes de prédication objectivée. Dans Copy \& Gournay (2009), l'analyse de quelques contextes discursifs où apparaissent typiquement les inversions locatives permet de 
En cas de défigement, cependant, le calcul des repérages dans l'énoncé ne se fait plus entièrement par rapport au proto-énonciateur. On le voit dans les exemples qui suivent et qui exemplifient différents degrés d'intégration d'une expression figée dans une énonciation dont les coordonnées sont spécifiques :

(8) Sophie grinned at Ginette and squeezed her hand. Ginette grinned back. All the girls - their friends - held hands and bowed low to the audience again. At that moment Sophie knew that a friend in need was a friend indeed. In fact, she knew that many friends in need were many friends indeed. (Sophie's Friend in Need, Norma Charles, 2004)

Dans l'exemple (8), le défigement porte en premier lieu sur la détermination temporelle: le prétérit indique que le système de repérage situationnel de l'énoncé proverbial inclut dorénavant le sujet-énonciateur de la Situation-origine de l'énoncé rapportant qui renvoie au sujet du verbe de la principale (Sophie). Cette intégration énonciative dans le discours de l'autre est par ailleurs le corollaire d'une appropriation cognitive des valeurs de vérité de la prédication proverbiale dénotée par le verbe $k_{n o w}{ }^{12}$. Dans la phrase suivante, l'intégration du proverbe est plus marquée encore puisqu'il y a une modification de la détermination nominale lorsque a friend devient many friends.

Dans l'exemple (6) cité plus haut et repris ici, le proverbe boys will be boys se trouve enchâssé dans du discours indirect libre (DIL) dont le repère temporel est marqué par le prétérit.

(6) Paul Curry was back, in jeans and a sweatshirt and with two large pizzas Mona had made and sent so boys could be boys for the night. (Grisham, J. Bleachers).

montrer qu'elles correspondent à une stratégie narrative d'effacement d'un point de vue subjectif spécifique au profit d'un point de vue trans-subjectif fortement stabilisé et identifiable aux coordonnées spatio-temporelles de la situation de l'événement. Cependant, le figement ne se situe pas au même niveau de la constitution de l'énoncé dans les deux cas, expressions figées et inversions locatives. Dans les expressions figées, le figement concerne le choix des notions instanciant la lexie et les opérations du niveau énonciatif et les opérations de défigement aboutissent à un changement de statut discursif de l'énoncé. Dans la construction à inversion locative, le figement a lieu au niveau du nœud prédicatif, d'où le terme de prédication objectivée, et toute tentative de défigement du nœud prédicatif aboutit à la formation d'énoncés agrammaticaux. On peut alors parler d'une forme de grammaticalisation du marqueur de figement.

12 Je remercie les participants à l'atelier de stylistique du congrès de la SAES 2010 dont les remarques et questions m'ont permis d'affiner cette analyse notamment en ce qui concerne l'importance du verbe introducteur. Une recherche dans GOOGLE (effectuée le 16.06.10) à partir de la suite «said that a friend in need was a friend indeed " donne en effet 0 occurrence alors que la suite « «said that a friend in need is a friend indeed » aboutit à 7000 occurrences (avec ou sans guillemets encadrant la phrase proverbiale). Les occurrences vérifiées montrent qu'il s'agit pour la plupart d'énoncés avec say au passif de type « it is said that a friend in need... ». En revanche la suite " a friend in need was a friend indeed » (2410 occurrences) fait apparaître, dans les cas de discours rapporté, les verbes introducteurs tell, believe, know, be (well) aware notamment. 
Ici, il s'agit de rapporter un contenu de parole, en l'occurrence celles prononcées par Mona et rapportées par Paul puis, incluses dans une narration à la troisième personne ${ }^{13}$. On constate alors deux rangs de repérage supplémentaires par rapport à un cas de proto-énonciation. D'une part, on a une modification de la détermination modale dans l'énoncé proverbial qui est la trace d'un repérage par rapport au sujet-énonciateur auquel renvoie le personnage de Mona lorsque l'auxiliaire WILL devient CAN. D'autre part, il y a une modification de la détermination temporelle avec l'adjonction du morphème de prétérit qui marque un repérage par rapport au sujet-énonciateur rapporteur, c'est-à-dire le personnage de Paul.

Ces modifications sont alors le signe d'une double intégration et d'une appropriation de l'énonciation proverbiale par des sujets-énonciateurs spécifiques ${ }^{14}$. Elles montrent également que les marqueurs présents dans le proverbe sont interprétés comme ils le sont dans la langue ordinaire, ce qui consacre l'appartenance de ces énoncés au domaine du discours. Enfin, elles montrent que, malgré tout, le sens de l'énoncé proverbial persiste dans l'interprétation et que les choix des sujets-énonciateurs tendent à être limités par ceux du proto-énonciateur. En effet, on voit en (6) par exemple que si la modification de la détermination modale est à mettre au compte d'un énonciateur spécifique, celle-ci se fait dans le cadre d'un paradigme établi en amont, celui des auxiliaires de modalité.

Ainsi, toute opération d'intégration dans un discours spécifique fait sortir l'expression figée du Thésaurus auquel elle appartient, tout en gardant cependant un lien fort avec son statut locutionnel dans la mesure où toute modification est entendue par rapport à l'énoncé initial.

En effet, le défigement suppose un double parcours interprétatif qui entraîne un double découpage morphosyntaxique : le premier qui correspond à une lecture synthétique et non compositionnelle, le suivant analytique qui correspond à une interprétation basée sur la valeur des marqueurs dans l'expression figée en tant qu'ils appartiennent à la langue ordinaire. Ce parcours interprétatif implique la contiguïté des deux lectures, ce qui fait que tout défigement convoque, de fait, l'expression figée.

Ainsi, dans Twice upon a time, on entend once upon a time et, en même temps que l'on signale un décalage, on renvoie au discours du conte et on oblige à revenir sur la valeur des marqueurs dans l'expression figée.

Dans les débuts de contes transcrits, on trouve des jeux sur les marqueurs typologiques qui relèvent du défigement. L'analyse des exemples du corpus

13 On note par ailleurs l'absence de THAT qui suggère d'autant plus que ces paroles sont bien celles du personnage de Mona qui renvoie au sujet-énonciateur origine.

14 Il y a également construction d'un troisième rang de repérage avec l'intégration dans la narration. 
montre de surcroît que ce travail énonciatif se fait typiquement avec les moyens spécifiques à chaque langue.

\section{Les énoncés formulaïques de débuts de contes : d'une situation à l'autre}

Dans les débuts de contes, le recours au défigement se fait de manière privilégiée dans les formulettes qui constituent la fiction secondaire du récit et qui en marquent la prise en charge par un conteur spécifique.

Les formulettes ont une fonction paradoxale dans le conte. En effet, elles sont le lieu de la prise de parole du narrateur-conteur qui s'exprime et commente en son nom les événements de l'histoire. Cependant, elles répondent à des schémas eux-mêmes assez stricts et répétitifs. L'analyse des exemples du corpus permet cependant de montrer que ces formulettes s'organisent à plusieurs niveaux autour d'une déconstruction des marqueurs typologiques de type once upon a time there was en faisant appel à des procédés propres au système linguistique de la langue concernée. Elles permettent de ce fait de mettre en évidence le rôle de ces marqueurs typologiques dans la mise en place de la situation initiale du conte, mais aussi dans l'inscription du discours du conte dans la langue.

\section{Marqueurs typologiques et mise en place d'une situation initiale}

Le rôle des marqueurs typologiques est de poser la situation initiale du conte dans l'espace du récit et d'introduire le(s) protagoniste(s) du conte dans l'espace narratif :

(9) There was a small house, three daughters and their mother. The two sisters thought themselves grand ladies but as for the youngest, they used to hide her in the coal-hole so that no-one would see her. ( $A$ Dictionary of British Folk-Tales part A Fairy Tales)

En (9), le marqueur typologique, réduit à son strict minimum, pose l'existence du lieu et des quatre personnages autour desquels s'organise l'histoire. La construction utilisée pour cela est typiquement la prédication d'existence ${ }^{15}$.

La compatibilité de cette construction avec le contexte de début de conte s'explique par le fait qu'elle permet de façon privilégiée, d'une part, de poser

15 La construction de prédication d'existence en there est la structure la plus représentée en début de conte (60\% sur un corpus de 200 contes). Il est par ailleurs intéressant de constater que, dans le corpus, le marqueur typologique once upon a time est assez rare et presque uniquement limité aux débuts de contes merveilleux, tout comme il était une fois en français. On peut supposer que, s'il est ressenti comme le marqueur typique, cela est dû au fait qu'il permet de poser de façon exemplaire la situation initiale du conte comme spécifique mais indéterminée. 
l'existence d'une occurrence de quelque chose dans un espace-temps spécifique dont les coordonnées spatio-temporelles peuvent rester indéterminées, comme c'est le cas en (9), et d'autre part, de marquer la prise en charge du récit par une entité subjective support de point de vue qui peut également rester indéterminée ${ }^{16}$.

Par ailleurs, le fonctionnement énonciatif de ces marqueurs typologiques, en tant qu'énoncés de particitation, permet à tout locuteur de s'en saisir, si l'on peut dire, dès lors qu'il décide de prendre en charge le récit et d'en proposer sa version. Cette particularité énonciative contribue à la construction d'une situation de contage posée comme unique mais également indéterminée.

C'est autour d'une remise en question de cette construction narrative, énonciative et linguistique que s'articulent les formulettes.

\section{Formulettes et Défigement}

À partir des exemples ci-dessous, on peut voir comment s'organisent, dans les formulettes, le défigement du marqueur typique et la déconstruction du discours stéréotypé du conte.

(10) There was a king long ago, and if there ever was, there often was and will be again. (Folktales of Ireland)

(11) A long, long time ago - if I were there then, I wouldn't be there now; if I were there now and at that time, I would have a new story or an old story, or I might have no story at all—there was a king and a queen in Ireland, and they were married. (Folktales of Ireland)

(12) Once upon a time and it wasn't in your time nor my time but it was in jolly good days, Jack left home. (A Dictionary of British Folk-Tales: Part A, Fairy Tales)

Les marqueurs typologiques permettent de construire d'une part la spécificité de la situation initiale du conte posée comme unique (once) et indéterminée (a time) en différenciation de la situation de contage (a long time ago, verbe au prétérit), d'autre part, celle de la situation de contage alors que la construction en there est la trace d'une prise en charge du récit par un conteur particulier ${ }^{17}$. Dans les formulettes, ces deux situations constitutives du récit s'entremêlent à partir d'un travail sur les paramètres qui les fondent.

16 Pour une étude de there existentiel en tant que marqueur de prise en charge par un sujet évaluateur support de point de vue, voir Copy \& Gournay (2009).

17 Dans Copy \& Gournay (2009), le lien entre prise en charge par un sujet-évaluateur et prédication d'existence est étudié dans les phrases de début de conte entre autres. Ce lien apparaît également dans d'autres contextes discursifs, notamment les descriptions de scènes de crime, dans une comparaison avec le fonctionnement des inversions locatives. 
- Spécificité et unicité de la situation initiale du conte

Dans la formulette en (10), le jeu de déconstruction porte sur la détermination adverbiale de la situation de l'événement dont les coordonnées sont alors dénotées par des adverbiaux à sens itératif. Le marqueur typologique est repris en tant qu'hypothèse et la relation prédicative fait donc l'objet d'une désassertion. Dans la protase, on trouve le marqueur de parcours ever ${ }^{18}$, qui est opposé, dans l'apodose à often et again qui marque l'inclusion de l'occurrence en cours dans une classe ${ }^{19}$.

Par ailleurs, le travail sur le nœud prédicatif modifie la nature de l'assertion : avec there was on est dans le domaine du certain, avec there will on est dans le domaine du non-certain. De ce fait l'occurrence en cours est liée à celles à venir.

- Spécificité et unicité de la situation du contage

En (11), c'est sur la spécificité de la situation de contage que porte le travail de défigement par le biais également de la détermination adverbiale (then / now) et d'un jeu sur la modalité de l'assertion (was / would / might) qui porte cette fois sur la figure du conteur auquel renvoie le pronom $I$.

- Dimension fictionnelle du conte $v s$ la dimension factuelle du contage

En (10) et (11), la formulette introduit un jeu sur la valeur du prétérit qui peut selon les contextes renvoyer à du temporel (there was a king long ago) ou du modal avec valeur d'irréel (if there was ever / if I were there then / I would / I might) qui permet de souligner le caractère commun du décrochage en cours. Et paradoxalement, c'est la situation de contage qui se trouve dans le champ de l'irréel.

En (12), la reprise de a time par your time joue sur la polysémie du mot time (fois, époque) et permet de mettre en rapport la situation de l'événement (fictionnelle) et la situation d'énonciation (factuelle).

Ainsi l'étude de ces formulettes montre également qu'elles fonctionnent à partir des stratégies semblables à celles employées dans les variations d'expressions figées et notamment, à partir d'un jeu sur le fonctionnement de la langue. Dans les exemples du corpus, ces jeux mettent en évidence le fonctionnement sous-jacent du langage ainsi que les spécificités d'une langue donnée. Au niveau lexical, les jeux de mots s'organisent autour des phénomènes de polysémie et de synonymie. Au niveau grammatical, les variations mettent en évidence l'organisation de la détermination de l'énoncé et

18 Dans une perspective énonciative, ever est analysé comme marqueur de parcours, c'est-à-dire qu'il effectue un parcours sur l'ensemble des occurrences possibles sans qu'aucune ne soit sélectionnée. Il est à ce titre particulièrement compatible avec les énoncés non assertifs.

19 La classe correspond ici à la définition qu'on lui donne dans la $T O E$, c'est-à-dire la totalité des occurrences effectives, possibles et fictives correspondant à un ensemble de propriétés donné. 
de la prédication. Au niveau des marqueurs, elles montrent le lien entre les différentes valeurs de ceux-ci et permettent de souligner l'existence d'une valeur centrale à chacun d'entre eux.

\section{Conclusion}

Les débuts de contes transcrits se caractérisent souvent par une alternance d'énoncés dont les coordonnées énonciatives ne sont pas exactement les mêmes. Cette alternance est la trace d'une autre alternance qui se situe au niveau de la prise en charge énonciative du récit: on a un va-et-vient entre d'une part, une source de type proto-énonciateur qui apparait typiquement dans les énoncés où les variations modales sont impossibles, et d'autre part une source subjective de prise en charge qui valide l'actualisation du récit. Ce jeu énonciatif apparaît clairement dans le travail effectué sur les marqueurs typiques de début de contes dont le caractère figé bloque a priori toute prise en charge spécifique. Par ailleurs, l'ensemble de ces jeux de mots laisse ainsi apparaître que, dans les expressions figées que sont les marqueurs typologiques, on a bien affaire aux marqueurs de la langue dans ce qu'ils ont d'essentiel et que c'est cette valeur qui fonde tout particulièrement le fonctionnement poétique du texte. Ainsi, en utilisant ce procédé du défigement, le conteur, souligne le sens des marqueurs et rappelle son adhésion aux valeurs que ces expressions véhiculent mais aussi, par sa prise de parole, consacre l'appropriation qu'il fait de la langue. 


\section{Bibliographie :}

ADAM, Jean-Marie, 2001. Les Textes, types et prototypes, 4e édition, Paris, Nathan Université.

Belmont, Nicole, 1997. «L'Écriture des contes », De la voix au texte, L'Ethnologie contemporaine entre l'oral et l'écrit, textes rassemblés et publiés par N. Belmont \& J-F Gossiaux, Paris, éd. du CTHS.

BRICOUT, Bernadette, 1992. Le Savoir et la saveur. Henri Pourrat et « Le trésor des contes », coll. Bibliothèque des idées, Paris, Gallimard.

BRIGGS, Katherine Mary (ed.), 1991. A Dictionary of British Folk-Tales in the English Language, Part A: "Folk Narratives \& Fairy Tales", London, Routledge.

Chuquet, Hélène, CHuquet, Jean \& GILBERT, Éric, 2006. Glossaire français-anglais de terminologie linguistique, Théorie des opérations énonciatives : définitions, terminologie, explications, document : www.sil.org/linguistics/glossary_fe/defs/TOEFr.pdf (lien consulté le 9 juillet 2009).

COPY, Christine \& GOURNAY, Lucie, « Locative Inversion In Discourse: a strategy of non-commitment », Discours, 5 2009, [En ligne], mis en ligne le 04 novembre 2009. URL: http://discours.revues.org/index7355.html. Consulté le 13 mai 2010.

De MAtTIA, Monique, 2000. Le discours indirect en anglais contemporain, approche énonciative, Aix-en-Provence, Publications de l'Université de Provence.

Delmas, Claude, 2004. «Fragment d'un discours culinaire», Contrastes, Paris, Ophrys.

DUFAYE Lionel, 2008, Théorie des opérations énonciatives et modélisation: cheminement d'une réflexion linguistique. Paris, Ophrys. (L'homme dans la langue), 25-34.

GOURNAY, Lucie, 2005. «Quand l'agencement est la trace d'une prédication 'objectivée' ", Analele Universitatii din Craiova, Série Langues et littératures romanes, numéro spécial, an IX, 2005, Craiova, editura UNIVERSITARIA, 65-74.

GOURNAY, Lucie, 2006. Approche énonciative des catégories de marqueurs, document de synthèse présenté en vue de l'Habilitation à Diriger des Recherches, Paris 7-Denis Diderot.

Gross, Gaston, 1996. Les Expressions figées en français ; noms composés et autres locutions, Paris, Ophrys.

GRUNIG, Blanche-Noëlle, 1997. «La locution comme défi aux théories linguistiques : une solution d'ordre mémoriel? », La locution entre langue et usages, Fontenay St Cloud, ENS édition, 225-240. 
HANOTE, Sylvie \& CHUQUET, Hélène. 2004 'Who's speaking, please?' Le discours rapporté, coll. Gramvoc, Paris, Ophrys.

KLEIBER, Georges, 2004. «Petite sémantique des proverbes avec une vue spéciale sur les proverbes métaphoriques », conférence du 29 octobre 2004 dans le cadre du séminaire LATTICE, École Normale Supérieure (La diffusion des savoirs de l'École Normale Supérieure), document disponible en ligne, http://www.diffusion.ens.fr/index.php?res=conf\&idconf $=459$ (consulté le 22 juin 2010).

MaIngueneau, Dominique, 2004. «Hyperénonciateur et 'particitation' », Langages 156, 111-126.

MISRI, Georges, 1987. Le Figement linguistique en français contemporain, thèse de doctorat, Université René Descartes (Paris V).

Moon, Rosamund, 1998. Fixed Expressions and Idioms in English, a corpus-based approach, Oxford, Clarendon Press.

NUnBERG, Geoffrey, SAG, Ivan \& WASOW, Thomas, 1994. «Idioms », Language 70, 3, September, 491-528.

O'sullivan, Sean (ed. and trans.), 1966. Folktales of Ireland, Chicago, The University of Chicago Press.

RASTIER, François, 1994. " Défigements sémantiques en contexte », La Locution entre langue et usages, Fontenay St Cloud, ENS édition, 308-331

Svensson, Maria-Helena, 2004. Critères de figement. L'identification des expressions figées en français contemporain, thèse de doctorat, Umeå, Umeå Universitet.

THURBER, James, 1939. « The unicorn in the garden », http://btflatt.tripod.com/stories/thurb1.htm (consulté le 22 juin 2010).

Virtanen, Tuija, 1992. Discourse Functions of Adverbial Placement in English: Clause-Initial Adverbials of Time and Place in Narratives and Procedural Place Descriptions, Turku, Åbo Akademi University Press.

Vuillaume, Marcel, 1990. Grammaire temporelle des récits, Paris, Minuit. 